\title{
Decay of correlations and invariance principles for dispersing billiards with cusps, and related planar billiard flows
}

\author{
Péter Bálint * Ian Melbourne ${ }^{\dagger}$
}

13 May 2008

\begin{abstract}
Following recent work of Chernov, Markarian, and Zhang, it is known that the billiard map for dispersing billiards with zero angle cusps has slow decay of correlations with rate $1 / n$. Since the collisions inside a cusp occur in quick succession, it is reasonable to expect a much faster decay rate in continuous time. In this paper we prove that the flow is rapid mixing: correlations decay faster than any polynomial rate. A consequence is that the flow admits strong statistical properties such as the almost sure invariance principle, even though the billiard map does not.

The techniques in this paper yield new results for other standard examples in planar billiards, including Bunimovich flowers and stadia.
\end{abstract}

\section{Introduction}

Lorentz gas models (and the associated discrete time billiard maps) are an important class of examples in mathematical physics. Their systematic study from the viewpoint of smooth ergodic theory was begun by Sinaŭ [30] in the 1970's. Sinaŭ focused on planar periodic Lorentz gases with convex obstacles (dispersing billiards) proving properties such as uniform hyperbolicity and ergodicity. The situation is analogous to the muchstudied case of Anosov and Axiom A systems for which advanced statistical properties such as decay of correlations and central limit theorems are by now classical [2, 28, 31]. In particular, if $\Lambda \subset M$ is a mixing hyperbolic basic set with Gibbs measure $\mu$ for an

\footnotetext{
*Institute of Mathematics, Budapest University of Technology and Economics, Budapest, Hungary, pet@math.bme.hu

${ }^{\dagger}$ Department of Mathematics, University of Surrey, Guildford GU2 7XH, UK, ism@math.uh.edu
} 
Axiom A diffeomorphism $f: M \rightarrow M$, then for all Hölder observables $v, w: \Lambda \rightarrow \mathbb{R}$ the correlation function

$$
\rho(n)=\int_{\Lambda} v w \circ f^{n} d \mu-\int_{\Lambda} v d \mu \int_{\Lambda} w d \mu,
$$

decays exponentially: $\rho(n)=O\left(\tau^{n}\right)$ for some $\tau \in(0,1)$. The corresponding results for billiards are complicated by the presence of singularities and unbounded derivatives, but these difficulties were eventually overcome by Young [33] and Chernov [5] who proved exponential decay of correlations for planar periodic dispersing billiards by constructing a certain type of infinite Markov extension called a Young tower [33, 34].

The situation for flows is considerably more subtle. Whereas mixing Axiom A diffeomorphisms always have exponential decay of correlations for Hölder observables, there exist Axiom A flows that are mixing but at an arbitrarily slow rate [29, 26]. Currently, it is not known if exponential decay of correlations is typical for Axiom A flows. However, Dolgopyat [14] (see also [16]) proved that typical Axiom A flows are rapid mixing (faster than any polynomial rate) for sufficiently regular observables. Melbourne [18, 19] extended this result to flows with a Poincaré map modelled by a Young tower, proving for example that the planar periodic Lorentz gas with finite horizon is rapid mixing. For this particular flow, Chernov [6] was able to prove stretched exponential decay of correlations.

Thus, generally speaking our understanding of planar periodic dispersing billiards and Lorentz gases is now as advanced as in the Axiom A case. However, there are a number of classes of planar billiards for which open questions remain [7, 9, 11]. In this paper, we focus mainly on questions related to decay of correlations for Lorentz gas flows, building on [18] (rapid mixing) and [19] (slow mixing).

Example 1.1 (Dispersing billiards with cusps) Dispersing billiards with cusps, where the boundary curves are all dispersing but the interior angles at corner points are zero, turned out to be much more involved technically than the usual Lorentz gases. For example ergodicity for the dispersing billiard map with cusps was only proved in the mid 1990's by Reháček [27]. By standard arguments, ergodicity implies K-mixing [9, Chapter 6] and the Bernoulli property [8, 24 for all the hyperbolic billiard examples.

Concerning rates of mixing, Chernov \& Markarian [10] proved, using the method of Young towers, that decay of correlations for the billiard map satisfies $\rho(n)=$ $O\left((\log n)^{2} / n\right)$. It was anticipated that the logarithmic factor is an artifact of the proof, and this factor was removed by Chernov \& Zhang [13] yielding the decay rate $\rho(n)=O(1 / n)$. It is believed that this result is optimal for the billiard map. This rate of decay of correlations is too weak for strong statistical limit laws (see [1]).

Since the collisions inside a cusp occur in quick succession, it is reasonable to expect a much faster decay rate in continuous time [11, Section 5.6], possibly even exponential decay of correlations. Exponential decay seems to be beyond current techniques even for simpler problems (such as the finite horizon planar periodic Lorentz 
gas). Previously, no results on decay of correlations for the flow (not even slow mixing) were available when there are cusps. In this paper we prove rapid mixing for the flow.

A byproduct of our proof is the almost sure invariance principle (ASIP) for the flow. We note that the ASIP implies numerous statistical limit laws such as the central limit theorem, the law of the iterated logarithm, and their functional versions, see for example [25, 21].

Example 1.2 (Bunimovich flowers) It was discovered by Bunimovich in the 1970's that billiard tables with focusing boundary components may also show hyperbolic behaviour. For the first examples of such tables, constructed for example in [3], the boundary components are either dispersing, or focusing arcs of circles, subject to some further constraints (see Section 3.1 below). Given their typical shape, such billiards are often called Bunimovich flowers. Later the probably best known example of a hyperbolic billiard with focusing boundary components, the Bunimovich stadium, was introduced in [4]. Focusing hyperbolic billiards have a rich literature by now it is worth pointing out Wojtkowski's contribution, [32 in particular, which clarified much of the mechanism behind hyperbolic behaviour. For further historical accounts, and detailed proofs of ergodicity, we refer to the monograph [9] and references therein.

As for rates of mixing, Chernov \& Zhang [12] show that in Bunimovich flowers the billiard map has decay of correlations $\rho(n)=O\left((\log n)^{3} / n^{2}\right)$. (Again, the logarithmic factor appears to be an artifact of the proof and it is expected that $1 / n^{2}$ is the optimal rate.) It follows that the map satisfies statistical limit laws such as the ASIP. See [20] for the scalar case and [21] for $\mathbb{R}^{d}$-valued observables.

By [23], the billiard flow immediately inherits the ASIP (and hence the other limit laws). However, previous results were unable to establish estimates for decay of correlations of the flow. We recall from [12] that the only effect slowing down the decay rate of the billiard map is sliding along a circular arc, where collisions occur in quick succession. Just as in the cusp example, it is reasonable to expect that the flow mixes better than the map in Bunimovich flowers.

In this paper, we prove rapid mixing for the flow.

Example 1.3 (Bunimovich stadia) Bunimovich [4] established hyperbolicity and ergodicity for the stadium billiard bounded by two parallel lines connecting two semicircles. Markarian [17] proved decay of correlations $O\left((\log n)^{2} / n\right)$ for the billiard map, and Chernov \& Zhang [13] improved this to obtain the optimal rate $1 / n$. This is too weak for strong statistical limit laws; indeed Bálint \& Gouëzel [1] prove a nonstandard limit law (nonstandard domain of attraction of the normal distribution with $\sqrt{n \log n}$ normalization) for typical observables. The same limit law holds for the flow [1, 23]. In particular, the ASIP fails for both the billiard map and the flow.

This time, we do not expect the flow to mix more rapidly than the map, but slow mixing does not follow from previous results. In this paper, we prove that the stadium flow decays at the same slow rate $1 / t$ as the map. 
Remark 1.4 Dolgopyat's results [14] on rapid mixing for Axiom A flows require the observables to be smooth in the flow direction. This restriction is inherited by the generalisation in [18, 19] to nonuniformly hyperbolic flows, and by the current paper. In particular, our results on rates of mixing for Lorentz gases do not apply to certain physically relevant observables such as position and velocity. At present, the only result on decay of correlations for Lorentz gas flows that includes general Hölder observables is Chernov's result for the planar periodic Lorentz gas with finite horizon [6].

On the other hand, our proof of the ASIP for billiard flows with cusps in Example 1.1 is valid for all Hölder observables.

The remainder of the paper is organised as follows. In Section 2, we recall background material on Young towers [33, 34], and on decay of correlations for flows possessing a Poincaré map modelled by a Young tower [18, 19]. In Section [3, we present our new results for Examples 1.1, 1.2 and 1.3.

\section{Young towers and flows}

Let $f: M \rightarrow M$ be a map, and let $h: M \rightarrow \mathbb{R}^{+}$be a roof function. Let $\phi_{t}: M^{h} \rightarrow M^{h}$ be the corresponding suspension flow. Thus the map $f(x)=\phi_{h(x)}(x)$ is a Poincaré map for the flow. Conversely, given $f$ and $h$ we define the suspension $M^{h}=\{(x, u) \in$ $M \times \mathbb{R}: 0 \leq u \leq h(x)\} / \sim$ where $(x, h(x)) \sim(f x, 0)$. Then the suspension flow is given by $\phi_{t}(x, u)=(x, u+t)$ computed modulo identifications.

In our examples, $M$ is a finite union of Riemannian manifolds. We will say that the map $f: M \rightarrow M$ is modelled by a Young tower if it satisfies the axioms introduced by Young [33, 34]. In particular, there is a set $Y \subset M$ with $\operatorname{Leb}(Y)>0$ that possesses an appropriate hyperbolic product structure. Furthermore there exists a measurable partition of $Y$ into countably many sets $Y_{i}$, and $r: Y \rightarrow \mathbb{N}$ with constant values $r_{Y_{i}}$ on the $Y_{i}$, such that the induced map $F=f^{r}: Y \rightarrow Y$ is smooth and uniformly hyperbolic when restricted to any $Y_{i}$, with respect to the hyperbolic product structure of $Y$. It is important to note that $r$ is not necessarily the first return time to $Y$, thus the corresponding tower map is a Markov extension of the original map $f: M \rightarrow M$. For further details of the construction see the original references.

For examples from billiards, there is a natural invariant measure $\mu$ (the Liouville measure) that is equivalent to Lebesgue measure on $M$ with $L^{\infty}$ density. In what follows we always assume that both the discrete return time $r$ (defined on $Y$ ) and the roof function $h$ (defined on $M$ ) are integrable with respect to Lebesgue measure and hence $\mu$. The natural flow-invariant probability measure $\mu^{h}$ on $M^{h}$ given by $\mu^{h}=\mu \times$ lebesgue $/ \int_{M} h d \mu$ coincides with Lebesgue measure for the billiard examples.

We aim to compute the rate of decay of correlations and statistical limit laws for sufficiently regular observables $v, w: M^{h} \rightarrow \mathbb{R}$. For $m \geq 0, \eta \in(0,1)$, the function space $C^{m, \eta}\left(M^{h}\right)$ consists of those $v: M^{h} \rightarrow \mathbb{R}$ for which $\|v\|_{m, \eta}=\|v\|_{\eta}+$ 
$\left\|\partial_{t} v\right\|_{\eta}+\cdots+\left\|\partial_{t}^{m} v\right\|_{\eta}<\infty$ where $\partial_{t}$ denotes the derivative in the flow direction and $\|v\|_{\eta}=|v|_{\infty}+\sup _{x \neq y, u \in[0, \max \{h(x), h(y)\}]}|v(x, u)-v(y, u)| / d(x, y)^{\eta}$.

We now summarize results from [18, 19] used in Section 3, Define

$$
\rho_{v, w}(t)=\int_{M^{h}} v w \circ \phi_{t} d \mu^{h}-\int_{M^{h}} v d \mu^{h} \int_{M^{h}} w d \mu^{h} .
$$

Theorem 2.1 ( [18] ) Let $f: M \rightarrow M$ be a map modelled by a Young tower and $h: M \rightarrow \mathbb{R}^{+}$be a Hölder roof function. Assume

- Exponential tails: $\operatorname{Leb}(y \in Y: r(y)>n)=O\left(\gamma^{n}\right)$ for some $\gamma \in(0,1)$.

Then typically the flow $\phi_{t}: M^{h} \rightarrow M^{h}$ is rapid mixing: there exists $\eta \in(0,1)$ and for any $n \geq 1$, there exists $m \geq 0, C \geq 1$, such that

$$
\left|\rho_{v, w}(t)\right| \leq C\|v\|_{m, \eta}\|w\|_{m, \eta} t^{-n}
$$

for all $v, w \in C^{m, \eta}\left(M^{h}\right)$ and all $t>0$.

Theorem 2.2 ( [19] ) Let $f: M \rightarrow M$ be a map modelled by a Young tower and $h: M \rightarrow \mathbb{R}^{+}$be a Hölder roof function. Assume

- Polynomial tails: $\operatorname{Leb}(y \in Y: r(y)>n)=O\left(1 / n^{\beta+1}\right)$ for some $\beta>0$, and

- $1 / h \in L^{\infty}$, or more generally there is an integer $p \geq 1$ such that $1 / h_{p} \in L^{\infty}$ where $h_{p}=h+h \circ f+\cdots+h \circ f^{p-1}$.

Then typically the flow $\phi_{t}: M^{h} \rightarrow M^{h}$ is polynomial mixing with rate $1 / t^{\beta}$ : there exists $\eta \in(0,1), m \geq 0, C \geq 1$, such that

$$
\left|\rho_{v, w}(t)\right| \leq C\|v\|_{m, \eta}\|w\|_{m, \eta} t^{-\beta}
$$

for all $v, w \in C^{m, \eta}\left(M^{h}\right)$ and all $t>0$.

Remark 2.3 In general, Theorems 2.1 and 2.2 hold typically subject to a nondegeneracy condition. However, this nondegeneracy condition is automatic for the billiards examples considered here because of the contact-like structure [19].

Remark 2.4 Theorem 2.1 implies that in situations where Young [33] obtains exponential decay of correlations for the billiard map $f: M \rightarrow M$, rapid decay of correlations holds for the flow $\phi_{t}$. Similarly, Theorem 2.2 implies that in situations where Young [34] obtains polynomial decay of correlations for the billiard map $f: M \rightarrow M$ with rate $O\left(1 / n^{\beta}\right)$, polynomial decay of correlations with rate $O\left(1 / t^{\beta}\right)$ holds for the flow $\phi_{t}$. 
Remark 2.5 The condition that $h$ is Hölder can be relaxed in Theorems 2.1 and 2.2. It suffices that $h$ is uniformly piecewise Hölder as follows. The billiard examples are examples of smooth dynamical systems with at most countably many singularities. Write $M=\bigcup M_{j}$ where the $M_{j}$ are the maximal subsets of $M$ on which $f$ is smooth. It is enough that $\sup _{j}\left\|h 1_{M_{j}}\right\|_{\eta}<\infty$.

By construction, the Young tower that models the billiard map "respects" the partition $\bigcup M_{j}$ in the following sense: as $\left.F\right|_{Y_{i}}$ is smooth, $\bigcup Y_{i}$ is necessarily a refinement of the restriction of $\bigcup M_{j}$ onto $Y$.

Let us make one more remark. Even though this is a very simple observation, this is the Key Observation behind the arguments of Section 3. Note that $M$ can be viewed as a Poincaré cross-section to the flow. It may be the case that there is an alternative cross-section $\widehat{M}$ with Poincaré map $\widehat{f}: \widehat{M} \rightarrow \widehat{M}$ (and roof function $\widehat{h}: \widehat{M} \rightarrow \mathbb{R}^{+}$) that can also be modelled by a Young tower and such that the tail distribution of the Young tower for $\widehat{f}: \widehat{M} \rightarrow \widehat{M}$ allows for a better control of the statistical properties. Note that the corresponding suspension flow $\widehat{\phi}_{t}: \widehat{M}^{h} \rightarrow \widehat{M}^{h}$ is identical to the original flow.

\section{Statistical limit laws}

Theorem 2.6 ( [21] ) Let $f: M \rightarrow M$ be a map modelled by a Young tower and $h: M \rightarrow \mathbb{R}^{+}$be a Hölder roof function. Assume

- Summable tails: $\operatorname{Leb}(y \in Y: r(y)>n)=O\left(1 / n^{\beta+1}\right)$ for some $\beta>1$.

Then the (vector-valued) ASIP holds: there exists $\lambda>0$, and for any Hölder observable $v: M^{h} \rightarrow \mathbb{R}^{d}$ with mean zero there is a d-dimensional Brownian motion $W$, such that (on a possibly enriched probability space)

$$
\int_{0}^{T} v \circ \phi_{t} d t=W(T)+O\left(T^{\frac{1}{2}-\lambda}\right) \text { as } T \rightarrow \infty \text { a.e. }
$$

Remark 2.7 Again, it suffices that the roof function $h$ is uniformly piecewise Hölder as in Remark 2.5. Similarly, the Hölder regularity requirement for the observable $v$ in

Theorem 2.6 can be relaxed. Define $V(x)=\int_{0}^{h(x)} v \circ \phi_{t} d t$. It suffices that $V: M \rightarrow \mathbb{R}^{d}$ is uniformly piecewise Hölder.

Remark 2.8 A more difficult question is the ASIP for the time-one map of a flow. A general argument of [15, 22] yields the scalar ASIP (for real-valued observables) in situations where (i) summable decay of correlations is established by certain techniques, (ii) the class of dynamical systems is closed under time reversal. This includes time-one maps for Axiom A flows [22]. Again the result is restricted to observables that are sufficiently smooth in the flow direction.

It is immediate from these considerations that the scalar ASIP holds for time-one maps of the flows in the cusp and flower examples (Examples 1.1 and 1.2). The vector-valued ASIP remains an open question even for time-one maps of Axiom A flows. 


\section{Billiard flows}

In this section, we specialise to billiard flows and treat the three examples discussed in the introduction. We begin with Example 1.2 (flowers) since this is the simplest. Then we treat Examples 1.1 (cusps) and 1.3 (stadia).

Let $Q$ be a compact domain in $\mathbb{R}^{2}$ with piecewise $C^{3}$ boundary $\partial Q$ and let us consider the corresponding billiard dynamics in $Q$ - the motion of a point particle that travels with unit speed and bounces off $\partial Q$ elastically (the angle of incidence is equal to the angle of reflection). The resulting flow $\phi_{t}$ is a three-dimensional volume preserving flow. There is a natural two-dimensional cross-section $M=\partial Q \times$ $[-\pi / 2, \pi / 2]$ corresponding to collisions, and the Poincaré map $f: M \rightarrow M$ is called the billiard map. We choose coordinates $x=(r, \varphi)$ on $M$, where $r$ (the position) is the arclength parameter along $\partial Q$ and $\varphi$ (the outgoing velocity) is the angle made by the reflected trajectory and the normal to the boundary. The natural invariant measure for the billiard map is is the Liouville measure $d \mu=\cos \varphi d r d \varphi$.

By components or arcs we mean the maximal connected $C^{3}$-smooth pieces of the boundary $\partial Q$. We use the same terminology for the corresponding (two-dimensional) components of the phase space $M$. We assume that the curvature of any component has a fixed sign, thus we may consider convex inwards (dispersing), convex outwards (focusing) and neutral arcs, and decompose the phase space as $M=M^{+} \cup M^{-} \cup M^{0}$, respectively.

The dynamics of the billiard map/flow may depend sensitively on further geometrical properties of $\partial Q$, including the types of the components present and their distances and angles. Below we restrict to certain physically relevant examples and cite the literature for their known dynamical properties. For further details on billiard dynamics in general, see [9] and references therein.

Our analysis relies on the standard fact that the billiard map $f$ and the roof function (collision time) $h$ are piecewise Hölder continuous. A simple argument shows that the Hölder exponent is $\frac{1}{2}$ for both $f$ and $h$. Note that $h(x)=|f(x)-x|$ so it suffices to verify that $f$ is $\frac{1}{2}$-Hölder.

It is clear that $f$ is smooth except near preimages of tangencies (grazing collisions) and writing $f=\left(f_{r}, f_{\varphi}\right)$ a standard calculation [9, Section 2.11] shows that $f^{\prime}$ is bounded except for singularities where it behaves like $1 / \cos \left(f_{\varphi}(x)\right)$. Writing $\psi=$ $\pi / 2-\varphi$ and suppressing the $r$-coordinates, we have $f_{\psi}^{\prime}(x) \sim 1 / \sin \left(f_{\psi}(x)\right) \sim 1 /\left(f_{\psi}(x)\right)$ so that $\left(\left(f_{\psi}(x)\right)^{2}\right)^{\prime} \sim 1$. Hence $f_{\psi}(x) \sim \psi^{1 / 2}$ which is $\frac{1}{2}$-Hölder as required.

\subsection{Bunimovich flowers}

Bunimovich [3] studied hyperbolicity and ergodicity for a class of billiard tables $Q \subset$ $\mathbb{R}^{2}$ such that (i) $M^{0}=\emptyset$ (no neutral components); the dispersing components $M^{+}$ may have arbitrary geometry, in contrast (ii) $M^{-}$consists of circular arcs all strictly smaller than a semicircle; (iii) if such a circular arc was extended to a full circle, this 
circle would be contained in $Q$; (iv) the neighbouring components are transversal (no cusps). Actually, the conditions can be relaxed and even neutral components can be allowed as long as certain technical assumptions are satisfied, see [12] for details.

Chernov \& Zhang [12] show that for Bunimovich flowers the billiard map $f$ : $M \rightarrow M$ can be modelled by a Young tower with tail estimate roughly $O\left(1 / n^{3}\right)$. It then follows from Young [34] that the map has decay of correlations roughly $O\left(1 / n^{2}\right)$. By [21], the vector-valued ASIP holds for Hölder observables for both the billiard map and the billiard flow.

However, there is no immediate conclusion for the decay of correlations of the flow. (Exponential tails are required in Theorem 2.1] while Theorem 2.2. would yield at best $O\left(1 / t^{2}\right)$ decay. In any case, the assumption that $h$ is bounded below in Theorem 2.2 is violated.) Nevertheless, we now prove rapid mixing for the flow for sufficiently smooth observables. (The scalar ASIP for the time-one map for the flow then follows from Remark 2.8.)

Theorem 3.1 Let $\phi_{t}$ be the flow corresponding to a Bunimovich flower. Then $\phi_{t}$ is rapid mixing (in the sense of Theorem 2.11).

Proof The argument in Chernov \& Zhang [12, p. 1546] demonstrates the existence of an alternative cross-section $\widehat{M} \subset M$ (with Poincaré map $\widehat{f}: \widehat{M} \rightarrow \widehat{M}$ ) that is modelled by a Young tower with exponential tails. We claim that the corresponding collision time $\widehat{h}: \widehat{M} \rightarrow \mathbb{R}^{+}$satisfies the uniformly piecewise Hölder condition in Remark 2.5. expressed of course in terms of $\widehat{M}, \widehat{f}, \widehat{h}$. Rapid mixing follows from Theorem 2.1.

First, we recall the definition of $\widehat{M}$ in [12]. As mentioned above we write $M=$ $M^{+} \cup M^{-}$where $M^{+}$corresponds to dispersing arcs and $M^{-}$corresponds to focusing arcs. Then $\widehat{M}$ is given by $\widehat{M}=M^{+} \cup E$ where $E \subset M^{-}$consists of only the first (sliding) collisions at each focusing arc, so $E=M^{-} \cap f\left(M^{+}\right)$.

Next we verify that $\widehat{h} \in L^{\infty}$. Since $\widehat{h}=h$ on dispersing arcs we can restrict attention to a single focusing arc $\Gamma\left(\subset M^{-}\right)$, and in particular the single first collision set $E_{\Gamma}=\Gamma \cap E$. Consider $x \in E_{\Gamma}$. There is an integer $n \geq 2$ such that $f^{j} x \in \Gamma$ for $j=1, \ldots, n$ and $f^{n+1} x \notin \Gamma$. Hence

$$
\widehat{h}=g+h \circ f^{n},
$$

where $g(x)=h(x)+h(f x)+\cdots+h\left(f^{n-1} x\right)$ is the amount of time it takes to "slide" along the $\operatorname{arc} \Gamma$. Hence $\left|\widehat{h} 1_{E}\right|_{\infty} \leq|\Gamma|+|h|_{\infty}$ and so $\widehat{h}$ is bounded.

Finally, we verify that there is a uniform Hölder constant for $\widehat{h}$ on each partition element. Again we may restrict to a single first collision set $E_{\Gamma} \subset \Gamma$ where $\Gamma$ is a focusing arc, but this time it is necessary to consider the finer partition $E_{\Gamma}=\bigcup_{n \geq 1} E_{n}$ where $E_{n}=\left\{x \in E: f^{j} x \in \Gamma\right.$ for $j=1, \ldots, n$ and $\left.f^{n+1} x \notin \Gamma\right\}$. (In other words, $\widehat{f}=f^{n+1}$ on $E_{n}$.) Note that the partition elements $E_{n}$ coincide with the smoothness components of $\widehat{f}: \widehat{M} \rightarrow \widehat{M}$, which is in agreement with Remark 2.5. 
Let $R$ denote the radius of the focusing arc $\Gamma$. Let $(r, \varphi)$ denote the standard coordinates and note that sliding occurs for $\varphi \sim \pi / 2$ so it is convenient to introduce $\psi=\pi / 2-\varphi$. It is immediate from the geometry of the circle that

$$
f(x)=(r+2 R \psi, \psi), \quad h(x)=R \sin \psi
$$

for all $x=(r, \psi) \in \Gamma \cap f^{-1} \Gamma$. Hence

$$
\left|g\left(x_{1}\right)-g\left(x_{2}\right)\right| \leq n R\left|\sin \psi_{1}-\sin \psi_{2}\right| \leq n R\left|\psi_{1}-\psi_{2}\right|
$$

for all $x_{1}, x_{2} \in E_{n}$. On the other hand, since the angle $\psi$ remains constant during the $n$ grazing iterations, it is elementary that there is a constant $C_{1}$ depending only on $\Gamma$ such that $C_{1} /(n+1) \leq \psi \leq C_{1} / n$ for all $x \in E_{n}$. Hence $\left|\psi_{1}-\psi_{2}\right| \leq C_{1}\left(\frac{1}{n}-\frac{1}{n+1}\right) \leq$ $C_{1} / n^{2}$ and so

$$
\left|\psi_{1}-\psi_{2}\right|^{\frac{1}{2}} \leq C_{1}^{\frac{1}{2}} n^{-1}
$$

Combining estimates (3.2) and (3.3), we obtain $\left|g\left(x_{1}\right)-g\left(x_{2}\right)\right| \leq C_{2}\left|\psi_{1}-\psi_{2}\right|^{\frac{1}{2}}$ where $C_{2}=R C_{1}^{\frac{1}{2}}$ depends only on the arc $\Gamma$.

In addition, since $f^{n}(r, \psi)=(r+2 n R \psi, \psi)$ on $E_{n}$ and $h \frac{1}{2}$-Hölder, $\mid h\left(f^{n} x_{1}\right)-$ $h\left(f^{n} x_{2}\right) \mid \leq C_{3}\left(\left|r_{1}-r_{2}\right|+(2 R n+1)\left|\psi_{1}-\psi_{2}\right|\right)^{\frac{1}{2}} \leq C_{4}\left(\left|r_{1}-r_{2}\right|+\left|\psi_{1}-\psi_{2}\right|^{\frac{1}{2}}\right)^{\frac{1}{2}} \leq$ $C_{4}\left(\left|r_{1}-r_{2}\right|^{\frac{1}{2}}+\left|\psi_{1}-\psi_{2}\right|^{\frac{1}{4}}\right)$. Combining the estimates for $g$ and $h \circ f^{n}$, we have shown that $\widehat{h}$ is Hölder on $E_{n}$ with constant independent of $n$ (and exponent $\frac{1}{4}$ ). Since there are only finitely many arcs, this completes the proof.

\subsection{Dispersing billiards with cusps}

Chernov \& Markarian [10] studied dispersing billiards with cusps, where the boundary curves are all dispersing - that is, $M=M^{+}$- but the interior angles at corner points are zero. By [10, 13], the billiard map $f: M \rightarrow M$ can be modelled by a Young tower with tail estimate $O\left(1 / n^{2}\right)$. It follows from Young [34] that the map has decay of correlations $O(1 / n)$. This is too weak for strong statistical limit laws (see [1]). Nevertheless we now prove rapid mixing and the ASIP for the flow.

Theorem 3.2 Let $\phi_{t}$ be the flow corresponding to a billiard table with cusps. Then $\phi_{t}$ is rapid mixing (in the sense of Theorem 2.1) and satisfies the ASIP (in the sense of Theorem [2.6).

Proof Following Chernov \& Markarian [10] we define $\widehat{M}$ by excluding a neighbourhood of each cusp. The new collision map $\widehat{f}: \widehat{M} \rightarrow \widehat{M}$ is modelled by a Young tower with exponential tails [10]. By Theorems 2.1] and 2.6, it again suffices to show that the new collision time $\widehat{h}: \widehat{M} \rightarrow \mathbb{R}^{+}$satisfies the uniformly piecewise Hölder condition in Remark 2.5. 
Consider a single cusp and let $E_{n}$ be the set of those points in $\widehat{M}$ that spend exactly $n$ iterates in the cusp before returning to $\widehat{M}$. Note again that, in accordance with Remark 2.5, the sets $E_{n}$ coincide with the smoothness components of $\widehat{f}$. The calculation in [10, p. 738] shows that $\widehat{h}$ is bounded on $E_{n}$ independent of $n$. Hence $\widehat{h}$ is bounded on the whole of $\widehat{M}$.

It remains to find a uniform Hölder constant for $\widehat{h}$ on each $E_{n}$. Explicit calculations are more difficult than in Section 3.1 so we search for coarser estimates. We claim that there are positive constants $\alpha_{1}, \alpha_{2}, \alpha_{3}, C_{1}, C_{2}$ (independent of $n$ ) such that

(i) $\left|\widehat{h}\left(x_{1}\right)-\widehat{h}\left(x_{2}\right)\right| \leq C_{1} n^{\alpha_{1}}\left|x_{1}-x_{2}\right|^{\alpha_{2}}$, and

(ii) $\left|\widehat{h}\left(x_{1}\right)-\widehat{h}\left(x_{2}\right)\right| \leq C_{2} n^{-\alpha_{3}}$,

for all $x_{1}, x_{2} \in E_{n}$. It then follows that $\left|\widehat{h}\left(x_{1}\right)-\widehat{h}\left(x_{2}\right)\right|^{\gamma+1} \leq C\left|x_{1}-x_{2}\right|^{\alpha_{2} \gamma}$ with $\gamma=\alpha_{3} / \alpha_{1}, C=C_{1}^{\gamma} C_{2}$, and so $\widehat{h}$ is uniformly piecewise Hölder as required, with exponent $\alpha_{2} \gamma /(\gamma+1)$.

We verify the claim with $\alpha_{1}=\frac{11}{6}, \alpha_{2}=\frac{1}{4}$ and $\alpha_{3}=\frac{1}{3}$ (and hence Hölder exponent $\left.\frac{1}{26}\right)$. Throughout, $C$ is a uniform constant that can change from line to line. Let $x_{1}, x_{2} \in E_{n}$. Recall that $f$ and $h$ are Hölder with exponent $\frac{1}{2}$. Furthermore, by [10, Proposition 4.1],

$$
\left|f^{j} x_{1}-f^{j} x_{2}\right| \leq C n^{5 / 3}\left|f x_{1}-f x_{2}\right| \leq C n^{5 / 3}\left|x_{1}-x_{2}\right|^{1 / 2}
$$

for $j=1, \ldots, n$. We deduce that

$$
\begin{aligned}
\left|\widehat{h}\left(x_{1}\right)-\widehat{h}\left(x_{2}\right)\right| & \leq\left|h\left(x_{1}\right)-h\left(x_{2}\right)\right|+\sum_{j=1}^{n}\left|h\left(f^{j} x_{1}\right)-h\left(f^{j} x_{2}\right)\right| \\
& \leq C\left|x_{1}-x_{2}\right|^{1 / 2}+C \sum_{j=1}^{n}\left|f^{j} x_{1}-f^{j} x_{2}\right|^{1 / 2} \\
& \leq C\left|x_{1}-x_{2}\right|^{1 / 2}+n C n^{5 / 6}\left|x_{1}-x_{2}\right|^{1 / 4} \leq C n^{11 / 6}\left|x_{1}-x_{2}\right|^{1 / 4}
\end{aligned}
$$

establishing estimate (i).

By [10, p. 738, last line], we have $h\left(f x_{j}\right)+\cdots+h\left(f^{n-1} x_{j}\right) \leq C n^{-1}$ for $j=1,2$. As shown in [10, pp. 748-749], the cell $E_{n}$ has diameter of order $n^{-2 / 3}$ so that $\left|h\left(x_{1}\right)-h\left(x_{2}\right)\right| \leq C\left|x_{1}-x_{2}\right|^{1 / 2} \leq C n^{-1 / 3}$. By time-reversibility of the construction, $\left|h\left(f^{n} x_{1}\right)-h\left(f^{n} x_{2}\right)\right| \leq C n^{-1 / 3}$. Altogether, we have $\left|\widehat{h}\left(x_{1}\right)-\widehat{h}\left(x_{2}\right)\right| \leq C n^{-1 / 3}$ establishing estimate (ii). (The estimates of [10] that we have used are established in the first instance for a special billiard table with three cusps, but then extended to the general situation in [10, Section 6].)

Remark 3.3 Again, we stress that the vector-valued ASIP for the flow holds for general (piecewise) Hölder observables, whereas rapid mixing is restricted to sufficiently smooth observables (as is the scalar ASIP for the time-one map of the flow which holds by Remark 2.8). 


\subsection{Bunimovich stadia}

Bunimovich 44 established hyperbolicity and ergodicity for the stadium billiard bounded by two parallel lines $\left(M^{0}\right)$ connecting two semicircles $\left(M^{-}\right)$. By [17, 13], the billiard map $f: M \rightarrow M$ can be modelled by a Young tower with tail estimate $O\left(1 / n^{2}\right)$. It follows from Young [34] that the map has decay of correlations $O(1 / n)$ for Hölder observables. This is too weak for strong statistical limit laws; indeed Bálint \& Gouëzel [1] prove a nonstandard limit law (nonstandard domain of attraction of the normal distribution with $\sqrt{n \log n}$ normalization) for typical observables. By [23], the same limit law holds for the flow. In particular, the ASIP fails for both the billiard map and the flow.

This time, we do not expect the flow to mix more rapidly than the map. Nor can we apply Theorem 2.2 directly since the roof function is not bounded below. Nevertheless the conclusion of Theorem 2.2 is valid.

Theorem 3.4 Let $\phi_{t}$ be the flow corresponding to a Bunimovich stadium. Then $\phi_{t}$ is polynomially mixing with rate $1 / t$.

Proof The argument in Chernov \& Zhang [12, p. 1548] demonstrates the existence of an alternative cross-section $\widehat{M} \subset M$ (with Poincaré map $\widehat{f}: \widehat{M} \rightarrow \widehat{M}$ ) that is modelled by a Young tower with exponential tails. However, the corresponding roof function $\widehat{h}: \widehat{M} \rightarrow \mathbb{R}^{+}$is unbounded, so Theorem 2.1 does not apply this time. As mentioned above, they show that the corresponding tower for $f: M \rightarrow M$ has tails decaying at the rate $O\left(1 / n^{2}\right)$. We note that the tower for $M$ is built over the same base $Y$ as the tower for $\widehat{M}$ but is strictly higher (since it incorporates the returns from $M$ to $\widehat{M}$ ).

Whereas the cross-section $\widehat{M}$ in 12 takes account of both sliding along circular arcs in $M^{-}$and bouncing between the parallel straight lines in $M^{0}$, we define an intermediate cross-section $\widehat{M^{\prime}}$ with $\widehat{M} \subset \widehat{M}^{\prime} \subset M$ that takes only account of sliding. That is, we define $\widehat{M}^{\prime}=M^{0} \cup E$ where $E=M^{-} \cap f\left(M^{0}\right)$ consists of the first (sliding) collisions in $M^{-}$.

It is immediate that the tower for $\widehat{f}^{\prime}: \widehat{M}^{\prime} \rightarrow \widehat{M}^{\prime}$ has tail decay rate no worse than the tower for $\widehat{M}$ : it shares the same base $Y$ as the other two towers, but is lower than the tower for $\widehat{M}$ (and higher than the tower for $M$ ). In particular the tower for $\widehat{M}^{\prime}$ has tail decay rate $O\left(1 / n^{2}\right)$.

Let $\widehat{h}^{\prime}: \widehat{M}^{\prime} \rightarrow \mathbb{R}^{+}$denote the new roof function. It remains to verify the hypotheses on $\widehat{h}^{\prime}$ in Theorem 2.2. The proof that $\widehat{h}^{\prime}$ is uniformly piecewise Hölder is identical to that in Section 3.1. We claim that $\widehat{h}_{p}^{\prime}$ is bounded below for $p=2$. Indeed, $\widehat{h}^{\prime}$ can approach zero only at points $x \in M^{0}$ which are close to one of the endpoints of a straight boundary component, that is, to the corner made with $M^{-}$, and have velocity almost tangent to the straight boundary component. But then $f x$ is about to undergo a long sequence of sliding collisions so that $\widehat{h}^{\prime}(f x)$ has magnitude approx- 
imately the length of a semi-circular arc in $M^{-}$. Hence $\widehat{h}_{2}^{\prime}$ is bounded away from zero as required.

Acknowledgements This research was supported in part by EPSRC Grant EP/D055520/1; by OTKA (Hungarian National Research Fund) grants: F 60206, TS 49835 and K 71693; and by the Bolyai scholarship of the Hungarian Academy of Sciences. We would like to thank the Budapest University of Technology and Economics and the University of Surrey for hospitality while part of this research was done. IM is grateful to the University of Houston for the use of e-mail.

\section{References}

[1] P. Bálint and S. Gouëzel. Limit theorems in the stadium billiard. Commun. Math. Phys. 263 (2006) 461-512.

[2] R. Bowen. Equilibrium States and the Ergodic Theory of Anosov Diffeomorphisms. Lecture Notes in Math. 470, Springer, Berlin, 1975.

[3] L. A. Bunimovich. The ergodic properties of billiards that are nearly scattering. Soviet Math. Dokl. 14 (1973) 1136-1139.

[4] L. A. Bunimovich. On the ergodic properties of nowhere dispersing billiards. Commun. Math. Phys. 65 (1979) 295-312.

[5] N. Chernov. Decay of correlations and dispersing billiards. J. Statist. Phys. 94 (1999) 513-556.

[6] N. Chernov. A stretched exponential bound on time correlations for billiard flows. J. Stat. Phys. 127 (2007) 21-50.

[7] N. Chernov and D. Dolgopyat. Hyperbolic billiards and statistical physics. International Congress of Mathematicians. Vol. II, Eur. Math. Soc., Zürich, 2006, pp. 1679-1704.

[8] N. Chernov and C. Haskell. Non-uniformly hyperbolic K-systems are Bernoulli. Ergodic Theory Dynam. Systems 16 (1996) 19-44.

[9] N. Chernov and R. Markarian. Chaotic billiards. Mathematical Surveys and Monographs 127, American Mathematical Society, Providence, RI, 2006.

[10] N. Chernov and R. Markarian. Dispersing billiards with cusps: slow decay of correlations. Commun. Math. Phys. 270 (2007) 727-758. 
[11] N. Chernov and L. S. Young. Decay of correlations for Lorentz gases and hard balls. Hard ball systems and the Lorentz gas, Encyclopaedia Math. Sci. 101, Springer, Berlin, 2000, pp. 89-120.

[12] N. I. Chernov and H.-K. Zhang. Billiards with polynomial mixing rates. Nonlinearity 18 (2005) 1527-1553.

[13] N. I. Chernov and H.-K. Zhang. Improved estimates for correlations in billiards. Commun. Math. Phys. 77 (2008) 305-321.

[14] D. Dolgopyat. Prevalence of rapid mixing in hyperbolic flows. Ergodic Theory Dynam. Systems 18 (1998) 1097-1114.

[15] M. J. Field, I. Melbourne and A. Török. Decay of correlations, central limit theorems and approximation by Brownian motion for compact Lie group extensions. Ergodic Theory Dynam. Systems 23 (2003) 87-110.

[16] M. J. Field, I. Melbourne and A. Török. Stability of mixing and rapid mixing for hyperbolic flows. Ann. of Math. 166 (2007) 269-291.

[17] R. Markarian. Billiards with polynomial decay of correlations. Ergodic Theory Dynam. Systems 24 (2004) 177-197.

[18] I. Melbourne. Rapid decay of correlations for nonuniformly hyperbolic flows. Trans. Amer. Math. Soc. 359 (2007) 2421-2441.

[19] I. Melbourne. Decay of correlations for slowly mixing flows. Proc. London Math. Soc. To appear.

[20] I. Melbourne and M. Nicol. Almost sure invariance principle for nonuniformly hyperbolic systems. Commun. Math. Phys. 260 (2005) 131-146.

[21] I. Melbourne and M. Nicol. A vector-valued almost sure invariance principle for hyperbolic dynamical systems. Ann. Probability. To appear.

[22] I. Melbourne and A. Török. Central limit theorems and invariance principles for time-one maps of hyperbolic flows. Commun. Math. Phys. 229 (2002) 57-71.

[23] I. Melbourne and A. Török. Statistical limit theorems for suspension flows. Israel J. Math. 144 (2004) 191-209.

[24] D. Ornstein and B. Weiss. On the Bernoulli nature of systems with some hyperbolic structure. Ergodic Theory Dynam. Systems 18 (1998) 441-456.

[25] W. Philipp and W. F. Stout. Almost Sure Invariance Principles for Partial Sums of Weakly Dependent Random Variables. Memoirs of the Amer. Math. Soc. 161, Amer. Math. Soc., Providence, RI, 1975. 
[26] M. Pollicott. On the rate of mixing of Axiom A flows. Invent. Math. 81 (1985) 413-426.

[27] J. Reháček. On the ergodicity of dispersing billiards. Rand. Comput. Dynam. 3 (1995) 35-55.

[28] D. Ruelle. Thermodynamic Formalism. Encyclopedia of Math. and its Applications 5, Addison Wesley, Massachusetts, 1978.

[29] D. Ruelle. Flows which do not exponentially mix. C. R. Acad. Sci. Paris 296 (1983) 191-194.

[30] Y. G. Sină. Dynamical systems with elastic reflections. Ergodic properties of dispersing billiards. Uspehi Mat. Nauk 25 (1970) 141-192.

[31] Y. G. Sină. Gibbs measures in ergodic theory. Russ. Math. Surv. 27 (1972) $21-70$.

[32] M. Wojtkowski. Principles for the design of billiards with nonvanishing Lyapunov exponents. Commun. Math. Phys. 105 (1986) 391-414.

[33] L.-S. Young. Statistical properties of dynamical systems with some hyperbolicity. Ann. of Math. 147 (1998) 585-650.

[34] L.-S. Young. Recurrence times and rates of mixing. Israel J. Math. 110 (1999) 153-188. 